\title{
Poor care and the professional duty of the registered nurse
}

Clair Gamble and Robin M. Ion

This is the author accepted manuscript. The final published version is available via doi:

http://dx.doi.org/10.7748/nop.2017.e908 


\section{Introduction}

This article explores the issue of poor nursing care delivered to older people. We consider poor care to involve acts of neglect, abuse or incompetence, which occur for any reason other than error (Ion et al., 2016a; Ion et al., 2016b). It is a significant issue in both the UK healthcare system (Roberts, 2016a) and internationally (Jonson, 2016; Reader \& Gillepsie, 2013).

Since the publication of the report into poor care at Mid Staffordshire NHS Trust (Francis, 2013) and the subsequent widely reported failures at Morecombe Bay (Walsh, 2013), the Vale of Leven (MacLean, 2014), and Winterbourne View (Phelvin, 2014), there can be few nurses who are unaware of the concerns that have been raised about standards in a range of UK health and social care settings, including those for older people. For example, in both Mid Staffordshire and at the Vale of Leven, many of those who suffered, and, in some cases died, were vulnerable older adults. It may be tempting to believe that this is a temporary phenomenon, resulting from local and transient stresses; an argument which implies that these problems might be readily resolved by attention to the specific local situation. For some, however, these cases are simply the most recent manifestation of care failures over a number of decades (Ryden Grange, 2015). In recent years, wide-ranging debate has explored why care failure in health settings happens and what might be done to prevent it. Two broad positions have been examined.

Firstly, poor care has been explained as an outcome of a combination of systemic problems including underfunding, managerialism, inadequate staffing levels and cultures in which the needs of the system are privileged above those of the individual patient; the underlying argument here is that failures in care are a consequence of factors outside the control of most registrants (Paley, 2014; Randall \& McKeown, 2014). The second explanation has focused on individual failing and has considered the personal qualities and moral position of practitioners (Roberts \& Ion, 2015; Darbyshire et al., 2015; Roberts 2016a \& 2016b). Readers with an interest in exploring these issues further are referred to Stenhouse et al. (2016) who have summarized much of this material. 
Whilst accepting the importance of these arguments, in this article we consider the problem from a different perspective. Drawing on the wider literature, and lessons learned from a range of settings, we consider some of the steps that might be taken to minimise risk of both systemic and individual failure in relation to vulnerable older adults. In doing this we hope to stimulate discussion and debate, and to provide suggestions for achievable, low, or no cost interventions to improve professionalism in the care of older people, and thereby reduce the potential for care failure. Concordant with Parker \& Conroy (2011) that general improvement in this area requires a whole workforce approach, which recognises complexity of need, we explore three particular issues. Firstly, we examine ageism and its potential negative influence on health care for older people. We argue that there is a need to create and maintain a culture which is respectful and empowering; through developing this, we believe that the chances of care failure occurring are reduced. Next, drawing on the recommendations of the Freedom To Speak Up report (Francis, 2015), we emphasise the importance of having in place, at both local and organisational level, robust policies for the reporting of concerns about care quality. Finally we consider the issue of individual accountability. While acknowledging the difficulties associated with challenging poor care, we argue that failure of individual registrants to address these places them in a potentially perilous situation which may constitute a dereliction of their commitment to the Code (Nursing and Midwifery Council (NMC), 2015).

\section{Care failure and the older adult}

It is now widely recognised that, over the next few decades, health care in the UK will face significant challenges in responding to the needs of an ageing population which will require more complex care, as a consequence of multiple morbidity. The care of older adults is therefore a priority area for most providers. For some people, the prospect of growing old and requiring increasing levels of support is frightening (Nilsson et al., 2000). This should perhaps not surprise us as our society is one which values independence and autonomy and is often fearful of the consequences of dependency, which is a common, but not universal, feature of the ageing process. This fear is likely to have been exacerbated where individuals have personal experience of poor care, and also by the series of reports published in recent years which have raised the possibility of a more widespread failure of care for older adults. 
Alongside Francis (2013), there is now a catalogue of reports which detail the unacceptable face of health care in some UK services. These include Dignified Care (Older People's Commissioner for Wales, 2011), which described care for some older people in hospital in Wales as 'shamefully inadequate’ and the 2011 Report of the Health Service Ombudsman (Health Service Ombudsman, 2011) which detailed ten cases of care failure across the English NHS.

More recently the report into care at the Vale of Leven hospital in Scotland (MacLean, 2014), noted both system wide and individual failures which contributed to the deaths of at least thirty-four patients, many of whom were elderly. In his summary of patient and relatives experiences at the hospital, Maclean raised concerns about failures in essential nursing care. Examples included: very poor standards of hygiene - despite the fact that C-Difficile infection was known to be present; problems with communication between staff, relatives and patients; perceived understaffing; poor staff morale; and an impoverished care environment.

Finally the Care Quality Commission (2014) reported on care provided to people living with dementia as they moved between the residential care setting $(n=129)$ and hospital $(n=20)$ in England. Although many examples of excellent nursing care were recorded, there was also considerable variation, leading to the claim that it was very probable that those living with dementia who required nursing input, or some form of social care would, at some point, encounter poor standards.

\section{Attitudes towards older people}

Key to our argument is the belief that positive attitudes toward older adults are central to the delivery of high quality nursing care. Unfortunately, ageism, the systemic or individual stereotyping of people on the grounds of age, is thought to be the most commonly experienced and institutionalised form of discrimination in the Western World (Age UK, 2011). It involves a complex manifestation of negative thoughts, beliefs, language and behaviours regarding older people which may have negative implications for health (Malta \& Doyle, 2016). Exposure to its effects is thought to reduce the chances of successful ageing (Centre for Policy on Ageing, 2009), which in turn is associated with increased morbidity and mortality (Blaine, 2013). This should be a concern in and of itself. Of equal, if not 
greater significance would be evidence that suggests ageism may have a direct impact on the care received by older people. Intuitively it may seem obvious that age discrimination will result in poor care, however, in their review of the literature, Kydd \& Fleming (2015) found the situation to be less clear cut in terms of a direct causal relationship. Despite this it has been argued that negative attitudes towards working with older people may contribute to difficulty in recruiting the best staff to work with this population (Kydd et al. 2013), while Kagan \& Melendez-Torres (2015) have argued that ageism has contributed to the relatively low priority given to research and innovation in the care and treatment of elders and the devaluing of work in the field; both of these issues may contribute to poor care experiences. Other practical effects of age related discrimination have been illustrated by Kagan (2008) in her analysis of ageism in cancer care, which noted that older people with cancer were at risk of under treatment and poorer care experiences which might, to some extent at least, explain their poorer survival rates. More recently, Schroyen et al. (2016) used vignettes and found that oncology nurses support for a range of intervention in cancer patients was lower for older patients than it was for younger ones. The fact that the negative attitudes of society toward the elderly are reflected in the nursing workforce should not surprise us, but it should certainly concern us.

One aspect of ageism which has gained increasing attention only in recent years is the use of derogatory terminology. Language which could patronise, belittle or insult our elderly population is in common use (Commission on Dignity in Care, 2012) and can cause harm parallel to that of racism or sexism (Nelson, 2016). Derogatory terminology in this context might range from neglecting to ask how someone would like to be addressed through to opting for socially acceptable, but patronising descriptors such as 'dear' or 'sweetheart' through to the use of phrases such as 'bed blockers', 'the demographic time bomb 'and 'the silver tsunami' (Tadd et al., 2011). Language of this type is unacceptable; it is not only derogatory but it is known to contribute to the risk of health problems being overlooked (Tadd et al., 2011). As such, on a very practical level, all nurses can play a part in addressing ageism by firstly attending to their own language through self-censoring of discriminatory terminology, but also by challenging its use by colleagues. It would be naïve to believe that language change alone could solve the problem of discrimination on the grounds of age, however, challenging 
the more blatant excesses of discriminatory terminology may at the very least promote a climate of reflection in which the NMC (2015) requirement that nurses prioritise people is more likely to be achieved.

\section{Reporting and responding to concerns about care quality}

One of the many very troubling findings of the inquiry into care at Mid Staffordshire NHS Trust (Francis, 2013) was that, while knowledge of the scale and detail of care failure was shared by many, at an organisational level there was a degree of detachment and inertia that allowed this state of affairs to continue. This is in direct contradiction of the Code (NMC, 2015), which makes it clear that nurses must prioritise the people in their care and take action when their safety is compromised. In order to minimise the risk of care failure, organisations must therefore have very clear policies in place to ensure that staff know how to raise and escalate any concerns they might have (Phair \& Heath, 2012). Reviews published by Ion et al. (2017), Bickhoff et al. (2015), and Jackson et al. (2014) confirm that the act of reporting concerns can be very stressful to the degree that some will chose to ignore the problem rather than face the potential negative consequences of whistleblowing. This should not surprise us; Francis (2015) in his Freedom To Speak Up Review described the experience of whistleblowing for some as:

'... a harrowing and isolating process with reprisals including counter allegations, disciplinary action and victimisation' (p10)

This indicates that knowing what to do and feeling able and empowered to enact this knowledge are different phenomena. The former requires a clear and unambiguous process which can be followed by the witness to poor care, while the latter requires that the whistleblower feels sufficiently safe to speak up. Francis (2015) describes five specific areas for intervention in order to improve the likelihood that concerns about poor care would be raised and dealt with in a supportive manner. Table 1 below summarises these. 


\begin{tabular}{|c|c|}
\hline Area for attention & Actions \\
\hline Promotion of a culture of safety & $\begin{array}{l}\text { Development of a 'just culture' in which employers work to en- } \\
\text { sure that, first and foremost, safety issues are addressed and where } \\
\text { blame and defensive approaches are minimised. } \\
\text { Learning and reflection are prioritised and valued by all. }\end{array}$ \\
\hline $\begin{array}{l}\text { Raising concerns should be nor- } \\
\text { malised }\end{array}$ & $\begin{array}{l}\text { Creation of a culture where speaking up is a normal and expected } \\
\text { behaviour } \\
\text { Investigation of concerns is treated as a priority with ultimate re- } \\
\text { sponsibility held at senior executive level. }\end{array}$ \\
\hline $\begin{array}{l}\text { Recognising the role of bullying } \\
\text { and on staff reporting behaviour }\end{array}$ & $\begin{array}{l}\text { Organisations should recognise and respond to the corrosive and } \\
\text { damaging impact of bullying and associated behaviour. This } \\
\text { should involve a zero tolerance approach supported by training of } \\
\text { managers and staff in terms of managing and responding to in- } \\
\text { stances of bullying. Training should recognise and respond posi- } \\
\text { tively to cases where bullying behaviour is a consequence of } \\
\text { work related stress }\end{array}$ \\
\hline $\begin{array}{l}\text { Organisational leadership which is } \\
\text { visible and accessible }\end{array}$ & $\begin{array}{l}\text { Leaders from unit to board level should take steps to be known. } \\
\text { Moreover they should work to ensure that staff find them profes- } \\
\text { sional and approachable. }\end{array}$ \\
\hline Staff should feel valued & $\begin{array}{l}\text { Where individuals raise concerns their professionalism should be } \\
\text { valued and acknowledged as a positive contribution to patient } \\
\text { safety. }\end{array}$ \\
\hline
\end{tabular}


If healthcare organisations are serious about addressing issues related to poor care for older people, it is our view that they should review their policies in light of the recommendations made by Francis (2015). With the right leadership, many of these recommended changes could be implemented at ward or unit level with relative ease and little or no cost. They do, however, require a willingness on the part of practitioners to raise their concerns and a commitment on the part of organisations and those at the most senior levels within them to listen and respond in an open and non-defensive manner.

\section{Personal accountability and poor care}

There is now a good deal of evidence to indicate that poor care sometimes goes unreported, often because staff are concerned about the personal consequences of raising concerns, but also because they sometimes perceive reporting as a futile activity which will have no meaningful outcome (Jackson et al., 2014; Prang \& Jelsness-Jørgensen, 2014; Bickhoff et al., 2015). Concerns about self and a belief that raising issues is futile are possible explanations for why some practitioners choose to take no action or become defensive when they encounter poor care. By way of example, Manuel \& Crowe (2014) clearly articulate the concerns of staff working in a complex environment and trying to balance responsibility to the patient with professional and also organisational accountability. Whilst fully acknowledging the difficulties which may face the individual who is witness to poor care, we contend that although these may explain behaviour, they do not excuse the failure to raise concerns about poor care. From a professional perspective, the Code (NMC, 2015) and NMC guidance on raising concerns (NMC, 2013) make it very clear that registrants must report; as with all other areas of practice, they are accountable for their actions and equally importantly their omissions in relation to poor care. This has been made clear recently by Roberts (2016a \& 2016b) who has argued that to suggest external factors, for example the fear of negative consequences, prevent the registrant from reporting care failure conflates a preference not to act with the inability to do so. In other words, registrants may decide to stay silent about poor practice for personal reasons; although this remains a personal choice, practitioners can and should choose to do the right thing, which in this case is to put aside any self-interest or self-doubt and prioritise the needs of the patient. It could, of course, be argued that this analysis 
simplifies a significant problem for some nurses. Although this may be true, it is also true that privileging of self over patients is likely to perpetuate sub-optimal care, jeopardise patient safety and, moreover, that it is a potential failure of accountability.

This line of argument also assumes that raising a concern will inevitably result in negative consequences. However, in their study of staff perceptions of whistleblowing, Jones and Kelly (2014) noted the potential for informal challenges of poor practice to have a positive impact on perpetrators. Recognising the potential trauma associated with formal whistleblowing, they draw attention to the everyday opportunities that exist to raise a concern with a colleague who, perhaps as a result of tiredness or stress has said or done something which is regrettable or unhelpful in relation to patient care, but which can be put right with relatively little or no threat to the perpetrator or the person raising the concern.

It would be naive in the extreme to assume that raising concerns is not, however, a potentially risky business; Francis (2015) has already made this very clear. Despite this, some nurses do choose to speak out. Ion et al. (2016) analysed student nurses accounts of responding to instances of poor care and noted that those who spoke up explained their actions by reference to moral and professional codes; their commitment to these appeared to provide them with the strength to stand up for what they felt to be right. Bickhoff et al. (2015) have also explored this issue, noting that students were more likely to speak out when they: i) strongly identified with the nurse as patient advocate role, ii) felt that they could expect support from key individuals such as mentors, and iii) had a clear sense that failure to do so might have significant negative consequences for patients. Under these conditions students felt more able to demonstrate the moral courage required to speak out in the face of poor care. Moreover, if nurses do not speak out in the face of poor care, then who will speak for the vulnerable older adult?

\section{Conclusion: what can be done to reduce the incidence of poor care delivered to older people?}

Care failure is a feature of health systems across the world. At its worst it is systemic and can result in large scale significant suffering and death as occurred at Mid Staffordshire NHS Trust (Roberts \& 
Ion, 2015). At an individual level it may often go almost unnoticed, except, of course, by those who experience it. As with all problems, there is no single solution to this issue. Better staffing levels and improved education are long term strategies which may well help. We have highlighted three other areas where attention could result in improvement to care and a reduction in poor care.

First, we have highlighted the unhelpful nature of everyday ageism. This is an area where practitioners can take personal action by examining their own prejudices and attitudes towards older people. Individuals can take the decision to eradicate ageist language from their professional vocabulary; to challenge it in colleagues from across the multidisciplinary team may be more difficult, but should not be impossible.

Second, organisations need to have guidance which clearly explains how staff can raise concerns about poor care. More importantly, however, these need to exist in an environment where speaking out is valued and welcomed. Directors of Nursing and Senior Charge Nurses can do much to create this culture, but it also needs to be valued by individual practitioners at all levels.

Finally, there will always be situations where the prospect of raising of a concern about poor practice will be threatening for those who witness it, and it is understandable, but not excusable, that they may consider turning a blind eye. As accountable professionals who have responsibility for the care of vulnerable older people, this temptation must be resisted. While organisations can and should create a climate in which speaking up is the norm, there will be occasions when the individual has to martial their own resources and show the moral courage which goes with role and responsibilty of the registrant. 


\section{References}

Bickhoff, L., Sinclair, P.M. \& Levett-Jones, T. (2015). Moral courage in undergraduate nursing students: A literature review. Collegian 0(0), http://dx.doi.org/10.1016/j.colegn.2015.08.002.

Care Quality Commission (2014). Online. Cracks in the pathway. Available at: http://www.cqc.org.uk/sites/default/files/20141009_cracks_in_the_pathway_final_0.pdf. (Accessed $3 / 8 / 16)$

Darbyshire, P., Ralph, N. \& Caudle, H. (2015). Editorial: Nursing's mandate to redefine the sentinel event. Journal of Clinical Nursing, 24, (11-12), pp. 1445-1446

Francis R. (2013). Report of the Mid Staffordshire NHS Foundation Trust Public Inquiry. The Stationary Office, England.

Francis R. (2015). Online. Freedom to Speak up: An Independent Review into Creating an Open an Honest Reporting Culture in the NHS. Available at: https://freedomtospeakup.org.uk/wpcontent/ uploads/2014/07/F2SU_web.pdf (Accessed 11/05/15).

Health Service Ombudsman (2011). Care and compassion: Report of the Health Service Ombudsman on ten investigations into care of older people.

Ion, R., Smith K., \& Dickens G (2017). Nursing and midwifery students' encounters with poor clinical practice: A systematic review. Nurse Education in Practice, (23), pp. 67 - 75

Ion, R., Jones, A. \& Craven, R. (2016a). Raising concerns and reporting poor care in practice. Nursing Standard, 31(15), pp. 55-62.

Ion R., Smith K., Moir J. \& Nimmo S. (2016b). Accounting for actions and omissions: a discourse analysis of student nurse accounts of responding to instances of poor care. Journal of advanced nursing, 72(5), pp. 1054 - 1064.

Jackson D., Hickman, L.D., Hutchinson, M., Andrew, S., Smith, J., Potgieter, I., Cleary, M. \& Peters, K. (2014). Whistleblowing: An integrative literature review of data-based studies involving nurses. Contemporary nurse 48(2), pp. 240-252.

Jonson, H.H. (2016). Framing scandalous nursing home care: what is the problem? Ageing and Society, 36(2), pp. 400-419

Jones, A. \& Kelly, D. (2014). Whistle-blowing and workplace culture in older peoples' care: qualitative insights from the healthcare and social care workforce. Sociology of health \& illness 36(7), pp. 986-1002.

Kagan, S.H. (2008). Ageism in cancer care. Seminars in oncology nursing 24(4), pp. 246-253.

Kagan, S.H. \& Melendez-Torres, G. (2015). Ageism in nursing. Journal of nursing management 23(5), pp. 644-650.

Kydd, A. and Fleming, A., (2015). Ageism and age discrimination in health care: Fact or fiction? A narrative review of the literature. Maturitas, 81(4), pp. 432-438.

Kydd, A., Toughy, T., Newman, D., Fagerberg, I. and Engstrom, G. (2014). Attitudes towards caring for older people in Scotland, Sweden and the United States. Nursing older people, 26 (2), pp. 33-40

MacLean R. (2014). The Vale of Leven Hospital Inquiry Report. Scottish Government: Edinburgh. 
Malta, S. \& Doyle, C. (2016). Mental health and well-being in older people: Butler's three constructs of ageism in Australasian Journal on Ageing. Australasian Journal on Ageing 35(4), pp. 232-235.

Manuel, J. \& Crowe, M. (2014). Clinical responsibility, accountability, and risk aversion in mental health nursing: A descriptive, qualitative study. International Journal of Mental Health Nursing 23(4), pp. 336-343.

Nelson, T.D. (2016). Promoting Healthy Aging by Confronting Ageism. American Psychologist 71(4), pp. 276-282.

Nilsson, M., Sarvimaki, A. \& Ekman, S., (2000). Feeling old: being in a phase of transition in later life. Nursing inquiry, 7(1), pp. 41-49.

Nursing and Midwifery Council (2015). Nursing and Midwifery Council Raising Concerns: Guidance for Nurses and Midwives. Available at: http:// www.nmc.org.uk/globalassets/sitedocuments/annual_reports_and_accounts/raising-concerns-30-march-2015-rebrand.pdf (Accessed 11/5/16).

Older People's Commissioner for Wales (2011). 'Dignified care?' the experiences of older people in hospital in Wales. Available at: http://www.olderpeoplewales.com/en/Reviews/dignity-and-respect/Hospital-review.aspx (Accessed 3/8/16).

Paley, J. (2014). Cognition and the compassion deficit: the social psychology of helping behaviour in nursing. Nursing Philosophy 15(4), pp. 274-287.

Parker, S.G. \& Conroy, S. (2011). Poor inpatient care for older people. BMJ: British Medical Journal 342, n/a.

Phair, L. and Heath, H. (2012). Safeguarding vulnerable older people in hospital. Nursing Standard, 27(4), pp. 50-55.

Prang, I.W. \& Jelsness-Jørgensen, L. (2014). Should I report? A qualitative study of barriers to incident reporting among nurses working in nursing homes. Geriatric nursing 35(6), pp. 441-447.

Roberts, M. \& Ion, R. (2015). Thinking critically about the occurrence of widespread participation in poor nursing care. Journal of Advanced Nursing 71(4), pp. 768-776.

Roberts, M. (2016a). A critical analysis of the failure of nurses to raise concerns about poor patient care. Nurs Philos. doi:10.1111/nup.12149

Roberts M. (2016b). Self-deception and poor patient care: A big idea from a Sartrean perspective. Nurse Education Today 46, pp. 1-3.

Schroyen, S., Missotten, P., Jerusalem, G., Gilles, C. \& Adam, S. (2016). Ageism and caring attitudes among nurses in oncology. International Psychogeriatrics 28(5), pp. 749-757.

Tadd, W., Hillman, A. Calnan, S., Calnan, M., Bayer, T. \& Read, S. (2011). Right place - wrong person: dignity in the acute care of older people. Quality in Ageing \& Older Adults 12(1), pp. 33-43.

World Health Organization (2015). Online. World report on ageing and health. Available at: http://apps.who.int/iris/bitstream/10665/186463/1/9789240694811 eng.pdf?ua=1 (Accessed 08/05/16). 